\title{
Statistical description of nonunimodality phenomena of electric breakdown voltages distribution of polymer coatings
}

\author{
Yu.P. Virchenko, A.D. Novoseltsev \\ Belgorod State University, 308015, Belgorod
}

Received November 22, 2020

\begin{abstract}
Multilayer enamel-lacquer polymer coatings are under consideration. For such physical objects the statistical model which permits to identify the physical cause of appearance of more than one peak of the electric breakdown voltages distribution function is constructed. Such an effect is connected with the fact that no more than one defect in cross section of each coating layer may appear to occur.

Keywords: air inclusions, independent random variables, unimodal distribution, dielectric strength, enamel-lacquer coating.

Statistical description of nonunimodality phenomena of electric breakdown voltages distribution of polymer coatings. Yu.P. Virchenko, A.D. Novoseltsev

Побудована статистична модель, яка дозволя виявити причину появи більш ніж однієї вершини у розподілі напруг електричного пробою багатошарового емаль-лакового полімерного покриття. Порушення одновершинності пов'язуться з тим, що в поперечному перерезі кожного шару покриття може перебувати не більш одного дефекту.

\footnotetext{
Сконструирована статистическая модель, которая позволяет выявить физическую ческого пробоя многослойного эмаль-лакового полимерного покрытия. В рамках модели нарушение одновершинности связывается с тем, что в поперечном сечении каждого слоя покрытия может находиться не более одного дефекта.
} причину появления более чем одной вершины в распределении напряжений электри-
\end{abstract}

\section{Introduction}

Investigation of electric breakdown including its theoretical analysis has a great history. In connection with this, see, for example, the monographs [1]-[6] where results of investigations on each historical period. From our opinion, despite the absence of new principal approaches to the study of this phenomenon, up to now, it is devoted the great attention for its investigation (see, for example, [7]-[9]). It is dictated by aspiration to theoretical understanding of this physical phenomenon and by the necessity of practical struggle with its consequences. At this, naturally, the most attention is devoted to the study of electric breakdown in solids which accompanied by degradation of their physical properties including the mechanical destruction.

Currently, we may analyze theoretically the electric breakdown of dielectric materials only by phenomenological representations, since consistent realization of such an analysis at microscopic level leads to extremely complicated constructions in frames of statistical physics. Thus, at the described situation, it is natural that theoretical analysis of electric breakdown in solid materials is based, as a rule, on rather rough models of general physics. Each of them is oriented for the description of one significant side of the phenomenon. Our work is devoted to theoretical analysis of the observable experimentally effect [10]. 
It represents the statistical non unimodality of random breakdown voltages of polymer enamel-lacquer coatings.

The proposed analysis is based on the simple general physics model that differs it from the work [11] (see also [13]) where an attempt of explanation of this effect is done using only some kind general property of small random samples of breakdown voltages characterizing of the experiment conditions where the effect is appeared. In frames of such an approach they cannot to detect the physical reason generated it. From our opinion it is not sufficient, since in theoretical physics, it is considered to be that the appearance of non unimodality should be connected with the presence of the definite physical mechanism generated it. On the basis of our proposed analysis, one may assert that the nonunimodality effect is the consequence of the specific space distribution of defects in the coating film.

\section{The phenomenology of electrical breakdown of enamel-lacquer coating}

The electrical breakdown is stipulated by ionization and/or by ties breaking between particles of dielectric material directly under action of electrical field. The dielectric strength $\mathrm{E}_{\mathrm{br}}$ of solid dielectrics relative to the electrical breakdown is the relation of breakdown voltage value of dielectric material to the layer thickness of the material in the direction of applied voltage. It lies within relatively narrow limits, namely, $100 \div 1000 \mathrm{MV} / \mathrm{m}$.

Presence of defects located by random way in the material and having some random sizes, stipulates, in general case, the random character of breakdown voltages. Statistical character of breakdown voltages $\tilde{E}$ is not essential in the case if sample geometric sizes essentially exceed geometric sizes of defects. It leads to smallness of statistical distribution dispersion.

Otherwise, in the case when the pointed out relation between sizes is not such great and the distribution dispersion differs in a noticeable way from zero, one may observe explicitly the statistical spread of breakdown voltages.

At the observation of majority of physical phenomena, for the measured physical values which have a statistical spread, such a situation is most common when histograms of values have the unique maximal channel. In mathematical statistics this property of histograms is named the unimodality. In connection with this they say about the unimodality of probability distributions describing these random variables. The absence of the unimodality property of statistical distribution points out the presence some kind special physical mechanism due to which the non unimodality of its probability distribution appears. Just such an unimodality violation of the breakdown voltages distribution observes when the measurement of the dielectric strength of enamel-lacquer coatings is done.

Let us consider a dielectric polymer enamel-lacquer coating. It has the form of multilayered film. The preparation technology of such coatings consists of the consistent application of some layers $3 \div 10$ of enamel-lacquer mixture dissolved in liquid organic solvent. The application of each layer is followed by its subsequent drying. The application of each layer accompanies the appearance of defects in the distribution of polymer substance which have random geometric sizes. These defects change the voltage of electrical breakdown of the layer at the place of their appearance and, correspondingly, they change the breakdown voltage of total multilayered coating. Such defects appear randomly during its preparation when new lacquer layer is applied. Defects represent air inclusions. They appear due to not predictable adhesion of small air bubbles to the surface of previous dried layer of the film during the application of the subsequent layer of the lacquer. It is essential that at the described physical scenario of such defects appearance there is only one air inclusion appears along the thickness of each new layer.

Thus, defects appearance is a result of pointed out technological process of the formation of film layers. Defects have the form which is similar the semisphere that is stipulated by surface tension of the bubble, if we do not account the wettability effect of air bubble with dry coating surface. At such a case each defect characterizes by one positive random variable, i.e. the bubble radius.

Availability of defects of described type in polymer coating reduces its electrical breakdown voltage since the air dielectric strength is significantly less than the dielectric strength of the coating material. Due to randomness of defect radiuses, the electrical breakdown voltage should be also a random variable. 
Therefore, results of experimental study of dielectric strength of described polymer coatings realized according to definite rule are fixed in the form of statistical histograms of breakdown voltages. On these histograms two maximums are observed [11].

It should be noted that, besides of described mechanism of appearing of random spread of measured electrical breakdown voltages, this randomness may be also as the consequence of randomness of defect centers distribution in each coating layer. Such a mechanism of randomness may be appeared experimentally in the case of small density of defects in each layer and finiteness of the size terminals by which the electrical voltage is applied to coating surface. Namely, if we denote average distance between defects in the fixed layer by means of $l_{0}$ and the size $l$ is the terminal size, then such a situation is possible if $l \lesssim l_{0}$. Possibility of terminal size influence on the appearance of the nonunimodal probability distribution has been studied in the work [12] (see also [13]) where the possibility of its appearing was investigated in probability distributions of maximums of small samples of independent positive random variables. It is shown that, despite on small sample volume of random variables that is the case when the relation $l / l_{0}$ is not some kind large value, the distribution of its maximum preserve the unimodality of the distribution of corresponding random variables.

In present work we build the model which permits to calculate the probability distribution of breakdown voltage and, in its frames, we give explanation of the nonunimodality appearance in histograms of experimentally observed electrical breakdown voltages in multilayered enamel-lacquer coatings. Such an result is strongly differed from theoretical predictions obtained in frames of statistical mechanics where the representation theorem is fulfilled.

\section{Statistical mathematical model}

Let there be the film of enamel-lacquer coating consisting of $N$ layers of equal thickness $d$ with the characteristic values $0.5 \cdot 10^{-1} \div 2 \cdot 10^{-1} \mathrm{~cm}$. We number the film layers by $m=1, \ldots, N$ in the order of their location in cross section of the film, for example, bottom-up.

The base of our statistical model, in frames of which we attempt to describe the appearance of breakdown in the in multilayered enamel-lacquer coatings are the random points $\left\{\tilde{x}_{l} ; l=1 \div N\right\}$ and the random variables $\tilde{r}^{(m)}>0, m=1 \div N$ connected with each random point $\tilde{x}_{m}$. The points $\left\{\tilde{x}_{l} ; l=1 \div N\right\}$ are the centers of semispheres on plane of each layer $l=1 \div N$ and variables $\tilde{r}^{(m)}$ are the random radiuses of semispheres corresponding to each of them. The average value $\langle\tilde{r}\rangle$ of them is equal to $3 \div 5 \cdot 10^{-2} \mathrm{~cm}$. Here, the number $m$ is the order number of the layer of the coating. If the point has the number $m=2, \ldots, N$ then it is located in the plane dividing planes with numbers $m-1$ and $m$. The point $\tilde{x}_{1}$ is located on the plane between the base and the coating.

Since each layer is prepared independently from others, it is natural to consider that the defects in different layers are also statistically independent to each other. Thus, we have $N$ copies of statistically equivalent point random fields being statistically independent which are numbered by the index $m=1 \div N$.

Since all defects are statistically independent, we consider that all random variables $\tilde{r}^{(m)}, m=1 \div N$ are independent and are equivalent to each other. We denote by $w(r)$ their common probability density.

Let $U$ be the dielectric strength of polymer material and $U_{0}$ be the dielectric strength of air. We represent the trajectory of electronic avalanche by which the electric breakdown is realized in the form of line consisting of subsequently connected segments. Each rectilinear segment connects two centers of defects located in neighboring layers of coating. The electrical breakdown voltage in the case when the electronic avalanche moves along the segment which connects the defect center in $m$ th layer to the defect center in $(m+1)$ th layer (see Fig.1) is formed either the dielectric strength of polymer material or the dielectric strength of air. On the basis of general physics reasonings, it is the sum of electrical breakdown voltage of $m$ th air bubble and electrical breakdown voltage of polymer material along the segment. The first summand is equal $U_{0} \tilde{r}_{m}$ according to the definition of the dielectric strength, and the second one is equal the electrical breakdown voltage of polymer material along this element, i.e. such a sum has the form $U_{0} \tilde{r}^{(m)}+U \cdot\left(\left|\tilde{x}_{m}-\tilde{x}_{m+1}\right|-\tilde{r}^{(m)}\right)$. 


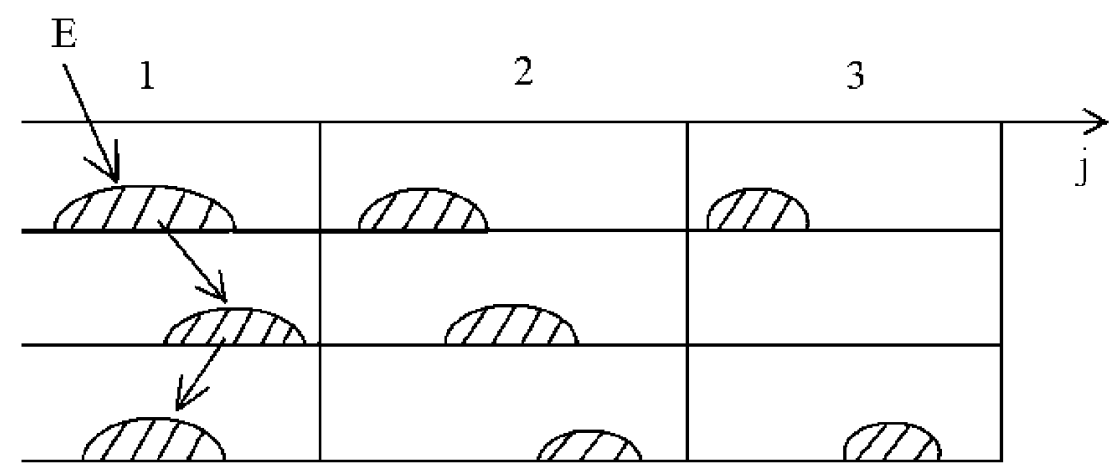

Fig. 1. It is shown schemetically three layers of the coating with defects embedded in them.

The movement the electronic avalanche along the trajectory is shown by arrows.

Then, in the case when the electronic avalanche is developed along the pointed out random trajectory, the electrical breakdown voltage is equal

$$
\sum_{m=1}^{N}\left[U_{0} \tilde{r}^{(m)}+U \cdot\left(\left|\tilde{x}_{m}-\tilde{x}_{m+1}\right|-\tilde{r}^{(m)}\right)\right] .
$$

We note that the avalanche is developed along the trajectory where this sum is minimal. By the way, the probability calculation of minimal trajectory realization is a particular problem in the percolation theory (see, the review [15]). It is clear that for realization of the electrical breakdown, it should be existed such a trajectory which is shown on the figure by arrows.

At this, the initial point $\tilde{x}_{1}$ and the ended one $\tilde{x}_{N+1}$ should be coved by terminals of electrical circuit with voltage applied to the polymer film. Thus, the random voltage $\tilde{E}$ of electrical breakdown connected with the random location of defects and their random sizes is given by the formula

$$
\tilde{E}=\sum_{m=1}^{N}\left[U_{0} \tilde{r}^{(m)}+U \cdot\left(\left|\tilde{x}_{m}-\tilde{x}_{m+1}\right|-\tilde{r}_{m}\right)\right]
$$

where the random sizes $\tilde{r}^{(m)}$ do not depend statistically on points $\left\{\tilde{x}_{l} ; l=1 \div N\right\}$ at each value $m=1 \div N$ and they are distributed identically with the density $w(\cdot)$. The problem consists of the determination of the density $f(E)$ of the probability distribution of this random variable if the probability distribution density $w(r)$ are fixed. At this, we consider that the average size $\langle\tilde{r}\rangle=\int_{0}^{\infty} r w(r) d r$ of the defect is much smaller than $l_{0}$.

Since the electronic avalanche that realizes the electrical breakdown is moved with great accuracy in a straight line, we may assert that, in the our model, with the same accuracy, the electrical breakdown voltage is determined completely by difference of electrical breakdown voltage of the polymer material without defects and electrical breakdown voltage of the air layer having the thickness equal to the sum of all sizes of air inclusions.

Let us introduce the value $E_{0}=U N d$ of electrical breakdown voltage of the polymer material and the positive difference $\nu=U-U_{0}$ of dielectric strengths of polymer and air. Then the probability $\operatorname{Pr}\{\tilde{E}<E\}$ of the electrical breakdown origin in the case when $\tilde{E}$ is less than some kind voltage $E$ reduces to the determining of the probability distribution of the sum $\tilde{r}=\sum_{j} \tilde{r}_{j}$ of independent and identically distributed variables

$$
\operatorname{Pr}\{\tilde{E}<E\}=1-\operatorname{Pr}\left\{\sum_{j=1}^{N} \tilde{r}^{(j)}<r\right\}
$$


where some $\tilde{r}^{(j)}$ may be equal to zero and $r=\left(E_{0}-E\right) / \nu$, since the inequality $E>E_{0}$. Consequently, the distribution density of the random variable $\tilde{E}$ is equal

$$
f(E)=\frac{d}{d E} \operatorname{Pr}\{\tilde{E}<E\}=\left.\nu^{-1} q_{N}(r)\right|_{r=\left(E_{0}-E\right) / \nu}
$$

Therefore, the unimodality violation of the probability distribution of electrical breakdown voltages of multilayered film is connected with the unimodality violation of the density $q_{N}(r)$. Applying the formula of the probability $\left(\begin{array}{l}N \\ k\end{array}\right)(1-v)^{N-k} v^{k}$ of $k$ successive events in consequence of independent random ones, where $v$ is the probability of defect appearance in one layer, we find

$$
\operatorname{Pr}\{\tilde{r}<r\}=(1-v)^{N} \theta(r)+\sum_{k=1}^{N}\left(\begin{array}{l}
N \\
k
\end{array}\right) v^{k}(1-v)^{N-k} \operatorname{Pr}\{\tilde{r}<r \mid k \neq 0\}
$$

where $\theta(\cdot)$ is the Heviside function defined as $\theta(r)=\{1$ at $r \geq 0 ; 0$ at $r<0\}$.

The last probability represents the probability distribution of the sum of $k$ independent identically distributed with the density $w(r)$ random variables. Therefore (see, for example, [16]), it is equal

$$
\operatorname{Pr}\{\tilde{r}<r \mid k \neq 0\}=\int_{0}^{r} \underbrace{(w * \ldots * w)}_{k}(x) d x,
$$

where the symbol $*$ denotes the binary operation of probability distributions convolution. In a result, the distribution density $q_{N}(r)$ is given, according to (1)-(2), by the following formula

$$
q_{N}(r)=(1-v)^{N} \delta(r)+\sum_{k=1}^{N}\left(\begin{array}{l}
N \\
k
\end{array}\right) v^{k}(1-v)^{N-k} \underbrace{(w * \ldots * w)}_{k}(r)
$$

where we take into account that $d \theta(r) / d r=\delta(r)$ is the Dirac function. Availability of $\delta$-functional singularity at the point $r=0$ in the distribution density $q_{N}(r)$ leads to the availability of $\delta$-functional singularity at the point $E=E_{0}$ of the distribution density $f(E)$. Then, for availability of the probability distribution nonunimodality of electrical breakdown voltages, it is sufficient to show that the distribution density $q_{N}(r)$ has, at least, one more peak at some kind point $r \neq 0$.

\section{Analysis of the statistical model}

In this section we show using the density $w(r)$ which has applied in [11] that the density $q_{N}(r)$ has the peak in nonzero point.

In practice, the type of the density

$$
w(r)=\frac{d}{d r} \operatorname{Pr}\{\tilde{r}<r\}, \quad \int_{0}^{d} w(r) d r=1
$$

and its parameters as well as the probability $v$ are not known. Therefore, at the theoretical analysis, it is necessary to operate with some kind model density.

In the model, proposed in the work [11], it was supposed that $w(r)=w_{0} e^{-\lambda r}$ at $\left.0<r<d, \lambda \sim\langle\tilde{r}\rangle\right\rangle 0$ and out of the segment $[0, d]$ the density $w(r)$ is equal to zero, where $w_{0}=\lambda\left(1-e^{-\lambda d}\right)^{-1}$. In this case, one may represent the formula of density in the form

$$
w(r)=w_{0} u(r) e^{-\lambda r}, \quad u(r)=\theta(d-r) \theta(r) .
$$

Substitution of the density (4) in (3) leads to the formula

$$
q_{N}(r)=(1-v)^{N} \delta(r)+e^{-\lambda r} \sum_{k=1}^{N}\left(\begin{array}{l}
N \\
k
\end{array}\right)\left(w_{0} v\right)^{k}(1-v)^{N-k} u_{k}(r), \quad u_{k}(r)=\underbrace{(u * \ldots * u)}_{k}(r),
$$


$k=2 \div N$ and $u_{1}(r)=\theta(d-r)$.

Now, we analyze the case $N=2$,

$$
u_{2}(r)=(u * u)(r)=\int_{0}^{r} \theta\left(d-r+r^{\prime}\right) d r^{\prime}= \begin{cases}r & \text { at } r<d, \\ \int_{r-d}^{d} d r^{\prime}=2 d-r & \text { at } d<r<2 d, \\ 0 & \text { at } r>2 d .\end{cases}
$$

Zero value is connected with the fact that inequalities $d+r^{\prime}>r$ and $r^{\prime}<d$ contradict to each other. Thus, the density $q_{2}(r)$, according to (5) at $r>0$, is given by the formula

$$
q_{2}(r)=\left[2\left(w_{0} v\right)(1-v)+\left(w_{0} v\right)^{2} r\right] e^{-\lambda r}
$$

if $r<d$ and, correspondingly,

$$
q_{2}(r)=\left(w_{0} v\right)^{2}(2 d-r) e^{-\lambda r}
$$

if $d<r<2 d$ and $q_{2}(r)=0$ at $r>2 d$. From these formulas, it follows that the density $q_{2}(r)$ decreases in the interval $r \in[d, 2 d]$, since $\dot{q}_{2}(r)<0$. At the point $r=d$ it has the negative jump equaled to $2\left(w_{0} v\right)^{2}(1-v) e^{-\lambda d}$. In the interval $r \in[0, d]$ it has the maximum:

1) at the point $r=d$, if $\dot{q}_{2}(r) \geq 0, r \in(0, d)$ when condition

$$
\begin{gathered}
w_{0} v-2 \lambda(1-v)-\lambda w_{0} v r>0, \\
1>\lambda d+2 \frac{\lambda}{w_{0}} \cdot \frac{1-v}{v}
\end{gathered}
$$

is fulfilled;

2) at an internal point of the interval $r_{*} \in(0, d)$,

$$
r_{*}=\frac{v w_{0}-2 \lambda(1-v)}{\lambda v w_{0}}
$$

when it is fulfilled the condition

$$
0<1-2 \frac{\lambda}{w_{0}} \cdot \frac{1-v}{v}<\lambda d
$$

3) at the point $r=0$ when the condition

$$
v w_{0} \leq \lambda(1-v)
$$

is fulfilled.

Thus, in frames of the model under consideration in two-layered film, the density $q_{2}(r)$ has the peak that is supplement to the $\delta$-functional one. Correspondingly, the density $f(E)$ has the peak that is supplement to the $\delta$-functional one in the point $E=E_{0}$. Therefore, the unimodality of the probability distribution of electrical breakdown voltages disappears. It is consistent with experimental data connected with electrical breakdown of multi-layered films of polymer coatings [11].

Let us analyze the case $N=3$. Calculate the function $u_{3}(r)$. It is defined by the convolution

$$
u_{3}(r)=\left(u_{2} * u_{1}\right)(r)=\int_{0}^{r} \theta(d-x) u_{2}(r-x) d x=\int_{0}^{r} \theta(d-r+x) u_{2}(x) d x .
$$

Then, the function $u_{3}(r)$ is equal

$$
u_{3}(r)=\int_{0}^{r} u_{2}(x) d x=\frac{r^{2}}{2}
$$


at $r<d$, since $\theta(d-r+x)=1$.

Correspondingly, we have

$$
u_{3}(r)=\int_{r-d}^{r} u_{2}(x) d x=\int_{r-d}^{d} x d x+\int_{d}^{r}(2 d-x) d x=\frac{3}{4} d^{2}-\left(r-\frac{3}{2} d\right)^{2}
$$

at $d<r<2 d$.

Further, we obtain

$$
\int_{r-d}^{2 d} u_{2}(x) d x=\int_{r-d}^{2 d}(2 d-x) d x=\frac{1}{2}(3 d-r)^{2}
$$

at $2 d<r<3 d$, since $2 d>r-d>d$.

At last, we have $u_{3}(r)=0$ at $r>3 d$.

In three-layered case the density $q_{3}(r)$ is defined at $r>0$ by three summands

$$
q_{3}(r)=\left[3\left(w_{0} v\right)(1-v)^{2} \theta(d-r)+3\left(w_{0} v\right)^{2}(1-v) u_{2}(r)+\left(w_{0} v\right)^{3} u_{3}(r)\right] e^{-\lambda r} .
$$

From this formula, on the basis of $(6),(7)$, we obtain

$$
q_{3}(r)=\left[3\left(w_{0} v\right)(1-v)^{2}+3\left(w_{0} v\right)^{2}(1-v) r+\left(w_{0} v\right)^{3} \frac{r^{2}}{2}\right] e^{-\lambda r}
$$

at $r<d$.

Further, we have

$$
q_{3}(r)=\left[3\left(w_{0} v\right)^{2}(1-v)(2 d-r)+\left(w_{0} v\right)^{3}\left(\frac{3}{4} d^{2}-\left(r-\frac{3}{2} d\right)^{2}\right)\right] e^{-\lambda r}
$$

at $d<r<2 d$ and we obtain

$$
q_{3}(r)=\frac{1}{2}\left(w_{0} v\right)^{3}(3 d-r)^{2} e^{-\lambda r}
$$

at $2 d<r<3 d$. At last, the density $q_{3}(r)$ is equal identically to zero at $r>3 d$.

From obtained formulas (9)- (10), it follows that the density $q_{3}(r)$ decreases at $r>2 d$ and it is equal to zero at $r>3 d$. We note that it is continuous at the point $r=2 d$ and it is equal $d^{2}\left(w_{0} v\right)^{3} / 2$. At the point $r=d$ the density has the negative jump, since, on the basis of $(9)$,

$$
q_{3}(d+0)=d\left(w_{0} v\right)^{2}\left[3(1-v)+\frac{1}{2} d\left(w_{0} v\right)\right] e^{-\lambda d}
$$

and

$$
q_{3}(d-0)=\left[3\left(w_{0} v\right)(1-v)^{2} d\left(w_{0} v\right)^{2}\left(3(1-v)+\frac{1}{2} d\left(w_{0} v\right)\right)\right] e^{-\lambda d},
$$

due to (8). Consequently, the negative jump is given by the formula

$$
q_{3}(d+0)-q_{3}(d-0)=-3\left(w_{0} v\right)(1-v)^{2} e^{-\lambda d}
$$

In connection with availability of the negative jump, the density has the peak in the interval either at its extreme points, if $\dot{q}_{3}(r) \neq 0$ or inside the interval in the opposite case. If it is realized such a case when the density $q_{3}(r)$ is not decreasing function inside $(0, d]$, this extremum is the peak of the density on $[0, \infty)$ being supplement to the peak at $r=0$. 


\section{Conclusion}

On the basis of general physics presentations, the statistical model has been built such that it permits to point out the possible physical reason of appearance of more than one peak in the distribution of electrical breakdown voltages.

Let us suppose that each layer may contain any arbitrary number of defects with statistically independent sizes, then mathematical analysis of the problem reduces to the scheme of independent variables sum. Probability distributions of such sums is unimodal when random variables have a common unimodal distribution. In particular, it tends to the gaussian distribution when the common distribution of summands has the density $w(r)(4)$ because of the probability of the empty layer is vary small.

In opposite case, let each layer may contain nonrandom number of defects which is small compared with the number $N$ being not large also. Then the probability to have a layer without the defect is not small. Such a summand gives an essential contribution to the distribution density $q_{N}(r)$. Moreover, in order to obtain the situation when the scheme of independent variables sum is not applied, we must suppose that the thickness $d$ should be much more than the average defect size. It is realized in the case under consideration taking into account the characteristic values $d$ and $\langle\tilde{r}\rangle$ represented in the section 3 .

Since there is no more than one defect may be located in one coating layer as one can see from experimental observation, the above described situation is realized in our model. Analysis shows that in such a situation the supplement peak may be appeared. Moreover, one peak of the density $f(E)$ necessarily coincides with the breakdown voltage $E_{0}$ of polymer material without defects. Due to this fact, we connect the nonunimodality phenomena of electrical breakdown voltages distribution with the fact of defect location pointed out.

\section{References}

1. A.P. Alersandrov, A.F. Valter, B.M. Vul, S.S. Gutin at al, Fizika dielektrikov, GTTI, Moscow (1932).

2. S. Whitehead, Dielectric breakdown of solids, Oxford University Press, Ney York 1951.

3. F. Walter, Dielektrischer Durchschlag, Handbuch der Physik. Hrsg. von S. Flugge. Bd. XVII, S.155, SpringerVerlag, Berlin (1956).

4. I.I. O'Dwier, The theory of electric conduction and breakdown in solid dielecrics, Clarendon Press, Oxford (1973).

5. M. Beyer, W. Boeck, M. Moller, W. Zaengl, Hochspannungstechnik. Theoretische und praktische Irundlagen, Springer-Verlag, Berlin (1986).

6. L. Solimar, D. Walsh, Lectures on the Electrical Properties of Materials, 4th ed., Oxford University Press, New York (1988).

7. E. Kuffel, W.S. Zaengl, J. Kuffel, High Voltage Engineering. Fundamentals 2d ed., Newnes, Oxford (2000).

8. G.A. Vorob'ev, S.G. Ekhanin, N.S. Nesmelov, Physics of the Solid State 47, 1083, (2005).

9. G.A. Vorobiov, Yu.P. Pokholkov, Yu.D. Koroliov, V.I. Merkulov, Fizika dielektrikov (Fizika sil'nych polei), TPU, Tomsk, (2003).

10. I.B. Peshkov, Electrotechnical materials, electric capacitors, wires, Nauka, Moscow, (1981). - 112 p.

11. R.P. Braginskii, B.V. Gnedenko, G.M. Zaitseva, S.A. Molchanov, Soviet Math. Dokl., 38, 501, (1989).

12. Yu. P. Virchenko, A.D. Novoseltsev, Journal of Physics: Conf. Series. 1479 (2020).

13. Yu. P. Virchenko, A.D. Novoseltsev, Belgorod State University Scientific Bulletin. Mathematics \& Physics, 51, 366, (2019).

14. Yu.P.Virchenko, O.I.Sheremet, Functonal materials, 7, 150, (2000).

15. M.F. Men'shikov, S.A. Mochanov, A.F. Sidorenko, Itogi nauki i tehniki, ser.teor.ver., mat.stat. i teor.kiber, VINITI, 24, 53, (1986).

16. B.V. Gnedenko, Kurs teorii veroyatnostei, Nauka, Moscow (1969). 